Documentation et bibliothèques

DOCUMENTATION BIBLIOTHEQUES

\title{
L'évaluation des services québécois d'information documentaire; plaidoyer pour une dose de réalisme
}

\section{Daniel Reicher}

Volume 24, numéro 3, septembre 1978

URI : https://id.erudit.org/iderudit/1055162ar

DOI : https://doi.org/10.7202/1055162ar

Aller au sommaire du numéro

Éditeur(s)

Association pour l'avancement des sciences et des techniques de la documentation (ASTED)

ISSN

0315-2340 (imprimé)

2291-8949 (numérique)

Découvrir la revue

Citer cet article

Reicher, D. (1978). L'évaluation des services québécois d'information documentaire; plaidoyer pour une dose de réalisme. Documentation et bibliothèques, 24(3), 107-112. https://doi.org/10.7202/1055162ar
Résumé de l'article

L'évaluation des services d'information documentaire se heurte à l'absence d'unités de mesure clairement définies. Pourtant, une évaluation doit être quantifiée pour que ses résultats soient utilisables. Après avoir souligné plusieurs écueils de l'évaluation qui rendent aléatoire l'étude de la satisfaction documentaire des usagers, l'auteur propose que cette difficulté soit résolue en mesurant la compétence des spécialistes en information documentaire plutôt que le contenu intellectuel ou physique des collections des services québécois d'information documentaire.
Tous droits réservés (c) Association pour l'avancement des sciences et des techniques de la documentation (ASTED), 1978
Ce document est protégé par la loi sur le droit d'auteur. L'utilisation des services d'Érudit (y compris la reproduction) est assujettie à sa politique d'utilisation que vous pouvez consulter en ligne.

https://apropos.erudit.org/fr/usagers/politique-dutilisation/ 


\title{
L'évaluation des services québécois d'information documentaire; plaidoyer pour une dose de réalisme
}

\author{
Daniel Reicher \\ Ecole de bibliothéconomie \\ Université de Montréal
}

\begin{abstract}
L'évaluation des services d'information documentaire se heurte à l'absence d'unités de mesure clairement définies. Pourtant, une évaluation doit être quantifiée pour que ses résultats soient utilisables. Après avoir souligné plusieurs écueils de l'évaluation qui rendent aléatoire l'étude de la satisfaction documentaire des usagers, l'auteur propose que cette difficulté soit résolue en mesurant la compétence des spécialistes en information documentaire plutôt que le contenu intellectuel ou physique des collections des services québécois d'information documentaire.
\end{abstract}

The evaluation of documentary information services comes up against the absence of clearly defined units of measurement. However, in order for it to be usable, an evaluation must be quantified. After having underlined several pitfalls of evaluation which render hazardous the study of the documentary satisfaction of users, the author proposes that this difficulty be resolved by measuring the competence of the specialists in documentary information, rather than the intellectual or physical contents of the collections of Quebec documentary information services.

La evaluación de los servicios de información y documentación se enfrenta con la ausencia de criterios bien definidos. Por otra parte, una evaluación debe ser cuantiada para que sus resultados puedan ser utilizados. Después de insistir en los numerosos escollos de la evaluación que hacen el estudio de la satisfacción de los usuarios aleatorio, el autor propone que esta dificultad esté resuelta mediendo la competencia de los especialistas de la documentación más que el contenido intelectual o físico de las colecciones de los servicios de información documental de Québec.

\section{Contexte}

Les difficultés de l'évaluation peuvent s'énoncer très simplement. II s'agit de trouver les moyens de: $1^{\circ}$ délimiter le phénomène que l'on veut évaluer, c'est-àdire mesurer; $2^{\circ}$ établir l'unité de mesure appropriée.

Nous tenterons d'abord d'illustrer ce que cerner un phénomène veut dire. Un service a évidemment pour mission de répondre à des besoins; mais, dans le cas d'un service d'information documentaire, s'agit-il de satisfaire les besoins des usagers ou ceux des documents, ceux du personnel ou ceux des fournisseurs?

Pourtant et uniquement afin de faire démarrer l'analyse de la question, nous ne retiendrons qu'une seule facette du phénomène, celle qui semble, de prime abord, la plus aisée à mesurer parce qu'elle traite d'un élément concret et inerte, le document. Notre objectif se limitant à l'exploration du cheminement d'une évaluation, nous nous contenterons d'une définition grossière du document comme étant une certaine quantité d'information mise "en conserve» sur un support documentaire. Voilà le phénomène délimité; il faut maintenant le mesurer à l'aide d'unités significatives.

Dans le système métrique, un litre d'eau pèse un kilogramme et occupe un décimètre cube. Trois unités pour mesurer trois facettes d'une même chose. Par analogie, pour évaluer le document, il faudrait donc s'entendre sur l'aspect du document que l'on veut mesurer. 
S'agit-il de son poids: veut-on savoir si le plancher de la bibliothèque est assez résistant pour le supporter?

S'agit-il de son épaisseur : veut-on déterminer le nombre de mètres linéaires de rayonnage qu'il faudrait aménager pour loger convenablement les documents?

S'agit-il de sa résistance aux conditions atmosphériques, de la quantité physique ou intellectuelle d'information qu'il emmagasine? S'agit-il enfin de l'impact de cette information sur l'usager? Vous remarquerez l'omission de la mesure la plus commune mais la plus mal définie de toutes: le nombre de volumes.

Autant d'aspects appelant probablement autant d'unités différentes de mesure. Cette simple énumération montre assez clairement que l'évaluation d'un service d'information documentaire ne peut se faire globalement sous peine d'en arriver à une réponse trop nébuleuse pour être significative.

Poussons notre argumentation plus avant, sans toutefois épuiser le sujet, car nous ne voulons, pour l'instant, que combiner deux aspects d'un même document. Un exemplaire $E$ d'un document $D$ logé dans la bibliothèque $B$ mérite-t-il une meilleure évaluation qu'un exemplaire $F$ du même document $D$ logé dans la bibliothèque $C$ ? La simplicité de cette question vous fera peut-être sourire, mais nous prétendons que c'est une question pertinente. En effet, le contrôle des conditions atmosphériques dans la bibliothèque $B$ peut être supérieur à celui qui prévaut dans $C$. Malgré l'égalité du contenu intellectuel, le contenant peut être en meilleur état et donc valoir davantage. Cette constatation prend tout son relief Iorsqu'on apprend qu'une portion importante des collections de la Library of Congress risque de tomber en poussière avant l'an 2000.

Par ailleurs, comment compare-t-on le contenant lorsque l'exemplaire $\mathrm{G}$ se trouve sur le support I et l'exemplaire $H$ sur le support $M$ ? En langage administratif, la bibliothèque $B$ qui possède $N$ documents sur support papier est-elle mieux ou moins bien préparée pour l'avenir que la bibliothèque $\mathrm{C}$ qui possède le même nombre $\mathrm{N}$ de documents sous forme de microtextes ou enregistrés dans la mémoire d'un ordinateur?

Ces quelques remarques nous permettent de revenir à l'énoncé du problème de l'évaluation tel que nous l'avons présenté au début de cet exposé. Tout programme d'évaluation est voué à l'échec à moins d'être basé sur une conception claire de ce que l'on veut mesurer, car il ne faut pas s'y tromper: seule la quantification peut permettre à un programme d'évaluation d'être objectif; seule l'uniformité des unités de mesure permet d'assurer des résultats universellement comparables.

Reprenons, de ce point de vue, notre exemple des divers exemplaires d'un même document logés dans des conditions atmosphériques différentes. A notre avis, une évaluation utile de ces exemplaires en tant que contenants devrait être basée sur une table de survie du document qui tienne compte des diverses variables atmosphériques: température, humidité, pollution, exposition à la lumière solaire, le tout mis en corrélation avec la nature physique et chimique du support documentaire. Ce n'est qu'en mesurant l'impact de ces facteurs qu'on arriverait à une évaluation objective de la probabilité de survie d'un document par ailleurs non utilisé. La solution devient évidente: l'analyse en laboratoire de la résistance du support documentaire soumis à des variations accélérées de chacun des facteurs atmosphériques énumérés. A cette table viendrait évidemment s'ajouter une table de résistance à l'utilisation basée, par exemple, sur la solidité de la reliure.

Avant de se lancer dans une campagne d'évaluation des services québécois d'information documentaire, il faudrait donc établir la liste des facteurs pertinents qui doivent être mesurés sans ambiguïté. Le but de cet exposé n'est pas d'établir cette liste mais bien plutôt d'alerter les amateurs à ce qu'une évaluation ne devrait pas être.

Après avoir examiné l'évaluation physi- 
que du document, il est peut-être temps d'étudier un exemple dont les réactions sont moins prévisibles. Considérons donc l'usager du service d'information documentaire. Toute enquête évaluative qui se respecte établit des statistiques de fréquentation enjolivées de rapports usagers / population qui fournissent des réponses à une multitude de questions culturelles. Faisons tout de suite retentir un signal d'alarme: les réponses passe-partout ne valent pas plus que les aspirines. On touche l'effet sans avoir décelé la cause. Si à une question, il peut y avoir plusieurs réponses correctes, la réponse qui convient à plusieurs questions doit être, elle, manipulée avec un scepticisme considérable. Par exemple, la fréquentation de la bibliothèque publique est un des facteurs utilisés pour situer le niveau culturel de la population. Or, le Québec a un taux de fréquentation des bibliothèques qui est actuellement de 18\%. Est-ce que les Québécois sont vraiment moins intéressés à la culture que d'autres groupes nationaux? $\mathrm{Ne}$ serait-ce pas davantage qu'ils sont d'origine latine plutôt qu'anglo-saxonne ou de religion catholique plutôt que protestante? Une réponse, trois questions possibles. On pourrait d'ailleurs affirmer, en se basant uniquement sur des statistiques, que les Québécois sont plus cultivés que les Français dont le taux de fréquentation des bibliothèques publiques n'est que de $9 \%$.

Reste à savoir si ces questions déclaratives sont bien formulées. On pourrait en douter lorsque l'on sait comment est calculé le taux de fréquentation. Que compte-t-on: le nombre d'entrées, le nombre de prêts? Penchons-nous sur le nombre d'entrées. Cette manière de calculer le taux de fréquentation est certainement utile pour établir des projections de besoins en espace des bibliothèques. Nous n'insisterons pas sur la nature des besoins satisfaits par le fait d'entrer dans un service d'information documentaire, car l'individu, indépendamment du motif de sa présence, ne serait-ce que pour se réchauffer, occupe tout de même le même espace. Le point que nous voudrions souligner touche au choix de l'unité de mesure. A notre connaissance, la fréquentation n'a jamais été mesurée en termes de personne/temps. Pourtant, si l'on veut calculer les besoins d'espace de lecture, pour savoir s'il est adéquat, il y a une différence notoire entre 50,000 entrées d'une durée totale de 25,000 personnes/heures et 30,000 entrées d'une durée de 60,000 personnes/heures. Intuitivement, les spécialistes en information documentaire en tiennent compte. Dans une bibliothèque universitaire, on se donne comme objectif un siège pour quatre étudiants; à la bibliothèque municipale, trois sièges pour mille habitants.

Malheureusement, des notions de prestige socio-mathématiques l'emportent trop souvent sur l'empirisme administratif. On célèbre l'acquisition du millionième volume comme s'il s'agissait d'une victoire sur l'ignorance. On manie des chiffres sans se soucier de leur pertinence et parce que les chiffres sont digitalement précis, on succombe à l'illusion que le phénomène luimême a, lui aussi, été décrit avec précision. Que celui qui n'a jamais été tenté de "désarrondir » un nombre pour qu'il fasse plus sérieux nous accuse d'avoir calomnié les administrateurs innocents.

Quant au nombre de prêts, nous voudrions faire son sort, une fois pour toutes, à l'illusion touchante créée par la notion de rentabilité de la documentation. Nous avons rencontré trop de rapports bibliothéconomiques où le fait de prendre un document sur une tablette pour le déposer dans une main humaine était considéré comme une transaction de nature rentable. Ceci peut être vrai chez un libraire, à condition toutefois que le client paie le document et que le vendeur ait correctement établi sa marge de profit. La transaction est alors commercialement rentable pour le libraire, mais nous ne pouvons concevoir qu'elle puisse l'être pour le client, à moins qu'il ne revende le document plus cher qu'il ne l'a acheté ou qu'il ne l'utilise pour une activité lucrative, telle l'emballage des pommes au marché.

On dérape malencontreusement vers une notion de rentabilité intellectuelle qui découle de l'expression américaine to sellan idea, mais qui ne se prête pas à la mensuration comptable. Pourtant, un rap- 
port aussi sérieux que celui de Clairoux ' sur la bibliothèque municipale de Montréal, en 1973, a succombé à la tentation de calculer la rentabilité du service du prêt dans les bibliothèques de quartier de cette ville.

Venons-en au point crucial du problème de l'évaluation.

\section{Débat}

Nous désirons savoir si nos services d'information documentaire fonctionnent bien. Pourquoi ? Serait-ce parce que le mot SERVICE nous tracasse? La question est importante car sa réponse déterminera la nature du type d'évaluation à élaborer pour aboutir à une mesure valable de nos activités.

Nous nous plaignons depuis longtemps que les statistiques que nous rassemblons avec soin et effort ne sont pas utilisées. II faudrait se demander si elles sont utilisables. Si nous négligeons de préciser, au préalable, les paramètres que nous voulons étudier, la confusion et l'inutilité triompheront.

En premier lieu, nous devrions définir ce que nous voulons mesurer: la capacité du service d'information documentaire à satisfaire l'usager ou la satisfaction réelle de l'usager. Les points de vue sont diamétralement opposés. Dans le cas de la première option, le spécialiste peut, au risque de manquer d'objectivité, établir la capacité du service; pour ce qui est de la seconde option, il faut admettre que seul l'usager peut dire s'il est satisfait, quels que soient nos doutes sur son aptitude à percevoir et analyser son sentiment de satisfaction.

Si vous avez fait votre choix d'une réponse à la première question, voici un deuxième casse-tête: quels outils documentaires le spécialiste en information documentaire doit-il prendre en considération lorsqu'il évalue sa capacité de satisfaire les besoins en information d'un usager? II peut mesurer l'information documentaire que son service possède, mais il peut aussi inclure toute l'information accessible par déplacement de la documentation ( location ou emprunt) ou par déplacement de

1. Jacques Clairoux. Etude sur l'organisation des bibliothèques municipales, Montréal, Ville de Montréal, octobre 1973, n.p. l'usager (entente de fréquentation).

La différence est de taille. Dans le premier cas, on mesure l'excellence des documents accumulés dans un endroit; dans le deuxième cas, on devra mesurer l'expertise administrative du spécialiste en information documentaire. A l'aube de la téléréférence, on peut se demander si l'évaluation du premier cas n'est pas en train de perdre tout son sens.

Ajoutons une troisième question. Le spécialiste bien formé, mais utilisant une collection documentaire médiocre a-t-il plus de mérite à satisfaire les besoins en information de l'usager que le spécialiste utilisant une collection excellente? Aux yeux du patron protecteur des bibliothécaires, oui, sans doute, mais nous doutons fort que l'usager se préoccupe du contexte pénible dans lequel fonctionne son informateur; il risque de prendre pour acquis que la capacité de recourir à une excellente source d'information constitue l'aspect le plus important de la compétence, peu importe le comportement du spécialiste, celui du bibliothécaire qui accumule des documents en abondance, ou celui du documentaliste qui maîtrise à fond l'exploitation des sources d'information. Or, jusqu'à présent, les formulaires d'évaluation se sont contentés d'accumuler entre autres questions innombrables des informations sur la quantité de documents, les supports documentaires, le taux de fréquentation, l'espace disponible.

Un formulaire était considéré comme mieux préparé s'il parvenait à raffiner ce type de questions... On doit se demander, en 1978, si l'on n'a pas mesuré des éléments qui ne peuvent pas apporter de réponse à la question essentielle, à savoir : les spécialistes québécois de l'information documentaire ont-ils la capacité de satisfaire leur clientèle?

Cette capacité est pourtant mesurable. Prenons comme exemple le cas qui semble inspirer le plus de crainte aux évaluateurs, car il touche directement l'usager, c'est-à-dire celui de la référence. Le spécialiste en référence ne peut manifester sa compétence que s'il parvient à établir un contact empathique avec l'usager. Or, d'autres professions, notamment celle des psychologues, affrontent le même problème et elles ont réussi à établir 
des instruments précis d'évaluation pour mesurer l'empathie en entrevue.

Le psychologue Conrad Lecomte utilise six comportements nettement différenciés pour mesurer les habiletés non verbales en entrevue 2 :

- position non face à face;

- contact brisé avec les yeux;

- posture fermée;

- éloignement de la personne en entrevue;

- état de teinsion;

- intonation discordante de la voix.

Chaque incident de comportement négatif est compté durant une période de temps donnée.

A l'aide d'une telle méthode, on peut donc quantifier la possibilité de transférer de l'information du professionnel au client. S'il est possible de quantifier un comportement qui a toujours été considéré par les bibliothécaires comme étant éminemment subjectif et qualitatif, nous avons bon espoir qu'il soit possible de mesurer précisément toutes les autres activités des spécialistes en information documentaire.

II faut bien se rendre compte de la signification de cette approche: à une question identique, le spécialiste qui fournit la réponse au client le plus rapidement est le plus compétent, peu importe qu'il dispose de meilleurs outils de travail, qu'il possède une culture plus vaste ou qu'il soit parvenu à établir de meilleurs contacts avec d'autres services d'information.

\section{En guise de conclusion}

Jusqu'à présent, les spécialistes en information documentaire ont axé, tant bien que mal, l'évaluation de leurs activités sur la documentation plutôt que sur le service effectivement reçu par l'usager ou tout au moins offert à celui-ci. Nous aboutissons ainsi à une situation assez paradoxale.

Nous comptons méticuleusement les

2. Conrad Lecomte, Grille d'évaluation d'habiletés non verbales, Document interne du Département de psychologie, Université de Montréal, 1978. monographies tout en déplorant, en même temps, dans certains domaines, qu'elles soient désuètes au moment où elles sont publiées, donc inutiles à l'usager.

Nous dénombrons soigneusement les abonnements de périodiques, souvent même les titres et, plus ou moins vaguement, les voiumes. Nous prétendons, en nous basant sur des constatations statistiques, que le fascicule d'un périodique n'est pratiquement plus consulté une fois relié. Dès qu'il a plus de dix ans, nous nous empressons de le cacher au plus profond de la bibliothèque.

Nous modifions les unités de mesure pour compter les rapports techniques, les publications officielles, les dossiers de presse, quand nous n'omettons pas purement et simplement de les compter, car nous devons bien admettre que les unités de mesure habituelles donneraient des résultats mathématiquement ridicules, surtout pour des documents que nous accusons d'être éphémères.

Le paradoxe est évident: vaut-il mieux compter des documents éphémères mais contenant de l'information encore à jour plutôt que des documents robustes mais déjà désuets au moment où nous les rangeons sur les rayons de la bibliothèque pour ne plus jamais les en déloger? Ne vaudrait-il pas mieux prendre pour acquis, dans l'évaluation des services, que la coopération est un but que nous atteindrons au plus vite afin de pouvoir satisfaire les besoins de l'usager par l'accès aux ressources globales du patrimoine bibliographique national ou même international?

Cette attitude nous permettrait de ne plus gaspiller notre énergie à compter nos outils, mais de nous concentrer plutôt sur l'évaluation de nos compétences bibliothéconomiques. Quand on évalue l'efficacité d'un réseau de santé publique, on se préoccupe beaucoup plus de connaître le nombre de médecins de diverses spécialisations que le nombre de bistouris ou le poids des médicaments disponibles.

II nous semble que le moment est venu d'adopter la même attitude pour notre domaine 
d'activité. Nous disposerions alors, enfin, des renseignements nécessaires pour décider si nos services sont potentiellement efficaces et si l'image que le public se fait de nos services est ou n'est pas conforme à la réalité.

Animé d'un esprit malin de contradiction, nous nous permettrons de terminer notre exposé en pourfendant un dernier moulin à vent.

Les spécialistes en information documentaire s'inquiètent énormément du manque d'intérêt que la population manifeste pour leurs activités. Cette inquiétude est parfaitement légitime si elle se fonde sur la peur de voir se tarir le marché du travail pour nous et nos successeurs dans la profession. Par réaction défensive, nous tentons de faire de l'animation sociale et de l'éducation permanente. En fait, ce que nous faisons est du loss leader marketing pour attirer le non-usager. Etant donné que ce dernier ne semble pas vouloir du type d'information que nous lui offrons, nous tentons, en fournissant autre chose, de faire fonctionner malgré tout le compte-tour du tourniquet de notre service. Pourtant notre mission consiste à fournir de l'information culturelle, sociale, scientifique et non pas à nous métamorphoser en garderie, en école ou en centre de service social. Nous avons déjà vu des bibliothèques-musées. Nous espérons ne pas voir la discothèque/audiovidéothèque devenir la discothèque/salle de danse. Non pas que nous nous objections à l'utilisation intensive des locaux de nos services si ceuxci sont par ailleurs trop peu fréquentés; mais nous éprouverions une réticence profonde à accepter de voir ces activités très méritoires, mais aussi très adventices, devenir partie intégrante de l'évaluation de nos services d'information documentaire.

Si nos services sont facilement accessibles - ce qui est encore loin d'être le cas -, si l'usager éprouve un besoin d'information - ce qui est instinctif chez l'être humain - et si nos spécialistes en information documentaire sont compétents, le non-usager deviendra un usager sans qu'on ait besoin d'attrappe-nigaud pour lui faire franchir la porte de nos services. vaincus de la nécessité de nous évaluer nousmêmes plutôt que d'évaluer nos collections, nous poserons, en guise d'ultime argument, la question-piège que nous destinons habituellement aux étudiants sceptiques: si vous deviez comparer l'efficacité de votre service d'information documentaire au service le plus efficace de la région de Washington, D.C., choisiriezvous la Library of Congress ou la C.I.A. ? Pensez-vous donc que la C.I.A. compte le nombre de documents que ses agents accumulent au lieu d'évaluer la compétence de ces derniers dont d'ailleurs dépend leur vie même? $\mathrm{Ne}$ devons-nous pas nous aussi évaluer l'aspect de nos services dont dépend notre survie professionnelle? 